\title{
La enseñanza remota no viene sin retos
}

\author{
Remote Teaching Doesn't Come Without Challenges
}

\section{Ensino remoto não vem sem obstáculos}

Mayra Almodóvar-López Hartnell College

California, Estados Unidos malmodovar@hartnell.edu

iD https://orcid.org/0000-0001-9921-775X

Julia Teresa Atiles

East Tennessee State University

Tennessee, Estados Unidos atiles@etsu.edu

iD https://orcid.org/0000-0001-6893-1942

Aleida Chavarría-Vargas Universidad Latina de Costa Rica

Heredia, Costa Rica aleida.chavarria@ulatina.cr

Dttps://orcid.org/0000-0003-4577-7262

Maria José Dias

East Tennessee State University

Tennessee, Estados Unidos diasma@etsu.edu https://orcid.org/0000-0002-3119-3883

Irma Zúñiga-León

Profesional independiente

Heredia, Costa Rica irmazunigaleon@gmail.com Dttps://orcid.org/0000-0001-7194-1877 
https://doi.org/10.15359/ree.24-S.15

https://www.revistas.una.ac.cr/index.php/educare

educare@una.ac.cr

Resumen: Se analizan retos que han enfrentado maestras de primera infancia; particularmente con infantes de 3 a 6 años, a raíz de la suspensión de servicios educativos presenciales debido a la COVID-19, así como las implicaciones que ha tenido en la formación y el desarrollo profesional.

Palabras claves: COVID-19; enseñanza remota; educadores primera infancia.

Abstract: Analysis of challenges faced by early childhood education teachers of 3 to 6 years old as a result of the suspension of face-to-face educational services due to COVID-19, and the implications in teacher education and professional development.

Keywords: COVID-19; remote education; early childhood educators.

Resumo: Análise dos desafios enfrentados por professoras da primeira infância (de 3 a 6 anos de idade) em decorrência da suspensão dos serviços educativos presenciais devido à COVID-19, e as implicações na formação docente e desenvolvimento profissional.

Palavras-chaves: COVID-19; ensino remoto; educadores da primeira infância.

Recibido: 09/09/2020 Aceptado: 05/10/2020

\section{Introducción}

Debido a la pandemia de la COVID-19, la población educativa ha vivido múltiples cambios en la forma como enseñamos a nuestros y nuestras estudiantes. El cambio repentino, del servicio educativo presencial al remoto, ha traído consigo retos a personas educadoras, alumnado, familias, instituciones educativas y comunidades. Nuestra reflexión tiene como objetivo identificar diversos desafíos enfrentados por las personas educadoras a cargo de niños y niñas con edades entre 3 y 6 años. Específicamente, en la perspectiva de su preparación y desarrollo profesional, la colaboración que se establece con los padres y madres de familia y el proceso de enseñanza-aprendizaje.

\section{Desarrollo}

El cierre abrupto de las instituciones educativas evidenció que personas educadoras de la primera infancia no estaban preparadas para lidiar con la continuidad del servicio educativo con la modalidad de enseñanza remota (Dias et al., en prensa). Como consecuencia de esta situación, surgió la necesidad de nuevos procesos de formación y desarrollo profesional, no solo en el uso de recursos tecnológicos de aprendizaje, sino también en la implementación de metodologías de educación remota. Esta educación debe proteger los aspectos establecidos por 
https://doi.org/10.15359/ree.24-S.15

la Convención sobre los Derechos del Niño en términos de salud, agua, saneamiento, nutrición, educación, entre otros (Alianza para la Protección de la Niñez y Adolescencia en la Acción Humanitaria, 2019) y los principios de la educación de calidad para este grupo etario. Entre estos se destacan el juego, el aprendizaje autónomo, el vínculo con familias y comunidades, las adecuadas interacciones sociales maestro-niño y niño-niño, los espacios físicos, las rutinas debidamente planificadas y la evaluación del aprendizaje por medio de la observación, entre otros (Copple y Bredekamp, 2009).

En la prisa de buscar maneras de educar a los niños y las niñas, las personas educadoras están enfrentado desafíos adicionales que oscilan desde escasez de alimentos en las familias, no contar con computadores, ni teléfono celular tanto las personas docentes como el alumnado, hasta fallas tecnológicas y falta de conectividad a internet. Al respecto, Rani (2020) y Román y Lentini Gilli (2020) expresan que la pandemia exacerba las condiciones existentes de pobreza e inaccesibilidad a la tecnología, que caracteriza a muchas familias alrededor del mundo. Por su parte, también Turkewitz y Villamil (2020) mencionan que, en las últimas dos décadas, la desigualdad en América Latina ha llegado al punto más elevado registrado en la historia.

Otra preocupación se refiere a la falta de comunicación con las familias del estudiantado, quienes, a su vez, están haciendo frente a la amplia gama de efectos económicos y de salud a raíz del brote de coronavirus (Goldstein et al., 2020). La pérdida de comunicación con familias trae consigo limitaciones para continuar con el proceso de enseñanza y aprendizaje en un periodo de desarrollo integral caracterizado por su trascendencia en la vida del ser humano (Campos, 2010; Devercelli, 2020). Estas circunstancias han llevado a las docentes a solicitar ayuda a otros padres, madres, familiares e integrantes de la comunidad, con el fin de continuar manteniendo el vínculo familia-institución. Así mismo, dado que algunas personas cuidadoras carecen de las habilidades educativas y conocimientos conceptuales para ayudar a los niños y las niñas; las personas educadoras, como resultado de la pandemia, han debido generar procesos de enseñanza y aprendizaje para adultos, adultas y jóvenes, sin contar con la preparación para favorecer sus procesos de enseñanza.

Las personas educadoras enfrentan otras dificultades vinculadas con el proceso de enseñanza-aprendizaje respecto a la disponibilidad de materiales didácticos (tales como libros, papel, crayones, tijeras, pinturas entre otros) y equipo tecnológico (computadoras personales, tabletas, impresoras, teléfonos, y conexión a Internet); razón por la cual, han debido utilizar sus propios materiales, adquirir equipos, y conexión a internet. En su compromiso profesional, generan material audiovisual (imágenes y videos) que publican en Facebook y WhatsApp, no obstante, sienten presión por tener que evidenciar el aprendizaje de sus discípulos (Dias et al., en prensa). Como algunas instituciones educativas se encuentran en lugares remotos, también las personas educadoras desarrollan materiales impresos para distribuirlos a cuidadores y 
https://doi.org/10.15359/ree.24-S.15

https://www.revistas.una.ac.cr/index.php/educare

educare@una.ac.cr

cuidadoras, ya sea poniéndolos a disposición para ser recogidos en centros educativos o enviándolos junto con paquetes de comidas para las familias; adicionando horas a su jornada laboral (Dias et al., en prensa).

Además, se agrega que la formación en servicio recibida por las personas educadoras oscila desde el uso de las herramientas tecnológicas básicas (apertura de cuenta de correo electrónico) hasta el empleo de plataformas de comunicación colaborativa; esto junto con un énfasis importante en el conocimiento y aplicación de protocolos de salud para el regreso a la educación presencial. No obstante, están ausentes temas relacionados con los procesos de enseñanza y aprendizaje remota, apropiados para el desarrollo integral de niños y niñas de 3 a 6 años. De manera, que estas actividades han respondido a las necesidades inmediatas como respuesta a la pandemia, mas no han profundizado en aspectos pedagógicos.

\section{Conclusiones}

La crisis generada por la COVID-19 ha puesto en evidencia la carencia de preparación profesional para atender los procesos de enseñanzay aprendizaje propios de la educación remota para niños y niñas de 3 a 6 años. Ahora, más que nunca, necesitamos repensar nuestros programas de preparación profesional y formación continua, con el fin de aprovechar las competencias desarrolladas durante la crisis. Es oportuno incorporar las habilidades que apoyen la enseñanza y el aprendizaje remoto promoviendo los principios de prácticas que caracterizan la educación en la primera infancia. Los planes de estudio renovados, a partir de los aprendizajes derivados de esta experiencia, deberán considerar nuevos contenidos que incorporen las características del desarrollo cognoscitivo, lenguaje, socioemocional y psicomotor de los niños y niñas de 3 a 6 años, en la atención y educación remotas. Así mismo, tanto la formación como la capacitación deben trascender los aspectos formativos para el trabajo con familias y comunidades, hacia la preparación para procesos educativos de carácter más complejo.

Nota general: La traducción y corrección de estilo han sido realizados por la autora y el autor.

\section{Referencias}

Alianza para la Protección de la Niñez y Adolescencia en la Acción Humanitaria (Marzo de 2019). Nota técnica: Protección de la niñez y adolescencia durante la pandemia del coronavirus (Versión 1). UNICEF. https://www.unicef.org/media/66276/file/SPANISH Technical

Campos, A. L. (2010). Primera infancia: Una mirada desde la neuroeducación. OEA; OEC. http:// www.iin.oea.org/pdf-iin/RH/primera-infancia-esp.pdf 
Copple, C. y Bredekamp, S. (Eds.). (2009). Developmentally appropriate practice in early childhood programs serving children from birth through age 8 (3. $\left.{ }^{\mathrm{a}} \mathrm{ed}.\right)$. Naeyc.

Devercelli, A. E. (2020). Supporting parents to support children's learning during COVID-19. World Bank Group. http://documents.worldbank.org/curated/en/277261597346869312/ Supporting-Parents-to-Support-Children-s-Learning-During-COVID-19

Dias, M. J. A., Atiles, J. T., Almodóvar, M., Chavarría Vargas, A. y Zúñiga León, I. (En prensa). Innovative early childhood teachers rise to the challenges brought about by COVID -19. Childhood Education: Innovations.

Goldstein, D., Popescu, A. y Hannah-Jones, N. (6 de abril, 2020). As school moves online, many students stay logged out. New York Times. https://www.nytimes.com/2020/04/06/us/ coronavirus-schools-attendance-absent.html

Rani, R. S. (22 de abril, 2020). Imagine online school in a language you don't understand. New York Times. https://www.nytimes.com/2020/04/24/learning/lesson-of-the-day-imagineonline-school-in-a-language-you-dont-understand.html

Román Vega, I., y Lentini Gilli, V. (Martes 21 de julio, 2020). Brecha digital y desigualdades territoriales afectan acceso a la educación. PEN. https://estadonacion.or.cr/brecha-digitaly-desigualdades-territoriales-afectan-acceso-a-la-educacion/

Turkewitz, J. y Villamil, S.(11 de julio, 2020). In Latin America, the pandemic threatens equality like never before. New York Times. https://www.nytimes.com/2020/07/11/world/americas/ coronavirus-latin-america-inequality.html 